\title{
Variable stars in the globular cluster M 92
}

\begin{abstract}
G. Kopacki
Wrocław University Observatory, Kopernika 11, 51-622 Wrocław, Poland

Received 1 December 2000 / Accepted 23 January 2001

Abstract. Results of a search for variable stars in the central region of the Oosterhoff II type globular cluster M 92 are presented. Out of the 28 variable and suspected variable stars listed in the Catalogue of Variable Stars in Globular Clusters (Clement 1997) only two were not observed. Surprisingly, almost half of the observed suspected variables did not show any evidence of variability. Only one out of the 11 candidate RR Lyrae variables of Kadla et al. (1983) appeared to be variable. Moreover, variable v7, until now classified as an RR Lyrae star with a period of about $0.515 \mathrm{~d}$, turned out to be of the BL Herculis type, with the period approximately twice that long. In addition, six new variables were found in the very core of the cluster: four of the RR Lyrae and the remaining two of the SX Phoenicis type. RRc variable v11 shows changes in the light curve shape. Due to the aliasing problems, however, we can state only that this star is biperiodic. There is a possibility that v11 belongs to the new group of recently discovered double-period RRc variables pulsating in non-radial mode. Altogether, light curves were obtained for 20 variable stars. The total number of known RR Lyrae stars in M 92 equals now 17, 11 of type RRab and the remaining $6, \mathrm{RRc}$. The mean period of RRab variables amounts to $0.63 \pm 0.05 \mathrm{~d}$, while the average period for RRc variables is equal to $0.36 \pm 0.05 \mathrm{~d}$. Mean $V$ magnitudes, mean $V-I_{\mathrm{C}}$ colours, and ranges of variations were derived for 12 RR Lyrae stars and the BL Herculis variable. Period-shift analysis for M 92 and M 2 resulted in finding that despite significant difference in metal abundance between these two clusters $([\mathrm{Fe} / \mathrm{H}]=-2.24$ for M 92 and -1.62 for M 2), there is no firm evidence for a shift in period.
\end{abstract}

Key words. stars: population II - stars: variables: RR Lyr - stars: variables: Cepheids globular clusters: individual: M 92

\section{Introduction}

This is the second paper in a series devoted to the CCD photometry of variable stars in globular clusters. In the first paper (Kopacki 2000) the results of the search for variables in M 53 were presented. Here we present the results of the photometric study of another cluster, M 92.

The extremely metal-poor $([\mathrm{Fe} / \mathrm{H}]=-2.24$ according to Zinn 1985) globular cluster M 92 (NGC 6341, C1715+432) is known to contain $13 \mathrm{RR}$ Lyrae variables with determined periods (Sawyer-Hogg 1973; Clement 1997). It is located relatively far from the galactic plane in the constellation of Hercules. In consequence, its reddening is small $(E(B-V)=0.023 \mathrm{mag}$ according to Schlegel et al. 1998) and the contamination by field stars is low. M 92 is one of the best studied globular clusters in the Galaxy. Many colour-magnitude diagrams of this cluster have been published. As far as we are aware, the most recent CCD photometric study of M 92 is that of Grundahl et al. (2000) and the newest colour-magnitude diagram has been provided by Rosenberg et al. (2000).

Send offprint requests to: G. Kopacki, e-mail: kopacki@astro.uni.wroc.pl
The newest version of the Catalogue of Variable Stars in Globular Clusters (CVSGC, Clement 1997) lists 28 variable and suspected variable stars in M 92. Hereafter, the numbering system of this catalogue will be used.

The first variable star in M 92, v11, was discovered by Woods in 1916 (Shapley 1922). Guthnick \& Prager (1925) detected the next 13 ones (v1-v10, v12-v14). Hachenberg (1939) determined the periods and the light curves for 13 variables and established that 12 of them were of the RR Lyrae type and one, v14, belonged to the W UMa group of eclipsing binaries. The latter star is probably not a member of the cluster. Out of the 14 already known variables, v13 was the only one he could not classify. The average magnitude of this star turned out to be consistent with those of other RR Lyrae stars in M 92, but due to very small range, comparable to the observational error, Hachenberg (1939) failed to derive a period. The next three suspected variable stars (v15 - v17; Nassau's numbers 12,15 , and 16 , respectively) were found by Nassau (1938). He supposed that except for $\mathrm{v} 7$ and v15 all variables in M 92 known at that time were of the RR Lyrae type.

There had been some confusion with designation of variables $\mathrm{v} 12, \mathrm{v} 15$, and $\mathrm{v} 16$. This is discussed in detail by 
Kadla et al. (1983). They also give the cross-identification of variable stars in the third catalogue of Sawyer-Hogg (1973) with numbering system of Nassau (1938). It appears now that v12 is Nassau's star no. 10 and v15 corresponds to Nassau's star no. 12.

Oosterhoff (1944) rediscussed the observations of Hachenberg (1939) and determined new elements for v8, $\mathrm{v} 11$, and $\mathrm{v} 12$, while for $\mathrm{v} 9$ he gave only an approximate period. Arp et al. (1953) did not notice any variations of v16 and stated the variability of v17 as uncertain. Walker (1955) searched for variable stars at the edges of the RR Lyrae instability strip and among the brightest red giants of M 92. His observations of v16 and v17 indicated that both stars were constant within 0.02 mag. For v13 (Nassau's no. 14) he detected variations, but was unable to derive a period. He supposed, however, that if the light variations are real, and if they are periodic, the period should be close to $0.3 \mathrm{~d}$. All 14 new RR Lyrae candidates he observed turned out to be constant, while three giants revealed scatter of the brightness large enough to be regarded as variables. These three suspected variable red giants, however, are not listed in the CVSGC.

Subsequently, ephemerides of several variables were redetermined by Kheylo $(1964,1965)$. The first study of the period changes of RR Lyrae stars in M 92 was done by Bartolini et al. (1968). These authors improved the periods of 10 variables, but could not find any periodicity for v15. Kukarkin \& Kukarkina (1980) studied the period changes of 10 RR Lyrae stars in M 92 using observations covering almost 50 years. They found two stars, v8 and v10, to show Blazhko effect (Blazhko 1907).

A search for variable stars in the core of M 92 has been done by Kadla et al. (1983). This work was restricted to the comparison of several photographic plates in the blink-comparator and resulted in the discovery of 11 new variables with amplitudes exceeding $0.3 \mathrm{mag}$. Because of their brightness, all these stars were supposed by Kadla et al. (1983) to be of the RR Lyrae type. These suspected variables were included in the catalogue of Clement (1997) with designations v18 - v28.

Almost 60 bright red giants in M 92, including also the three ones suspected to be variable by Walker (1955), were checked for variability by Welty (1985). All turned out to be constant in light. A search by Shara et al. (1994) for eruptions of dwarf novae in M 92 revealed no example of this phenomenon.

Recently, M 92 and its RR Lyrae stars were observed and studied also by Carney et al. (1992), Storm et al. (1992a, 1992b), Cohen (1992), and Cohen \& Matthews (1992). Storm et al. (1994) determined luminosities for two RR Lyrae variables, v1 and v3, from a Baade-Wesselink analysis.

In this paper we present the results of our search for variable stars in the central region of the globular cluster M 92 using the Image Subtraction Method of Alard \& Lupton (1998). We comment on variability of all stars listed in the CVSGC and for all stars proved by us to be variable we determine periods and plot the light curves.
We discuss also some results derived from the photometry of RR Lyrae stars in M 92.

\section{Observations and reductions}

The CCD observations presented here were carried out at the Białków station of the Wrocław University Observatory with the same equipment as that described by Kopacki \& Pigulski (1995). Two $6 \times 4 \operatorname{arcmin}^{2}$ fields of view, one centred approximately on the core of M 92 and the other shifted about 2.5 arcmin north and 2.6 arcmin east, were observed through $V$ and $I_{\mathrm{C}}$ filters of the Johnson-Kron-Cousins $U B V(R I)_{\mathrm{C}}$ system.

The observations were carried out in two seasons: on 9 nights between 1998 July 20 and September 1, and on 17 nights between 2000 May 4 and June 20. During the 1998 season only a few frames of the second field were taken. The main field, centred on the core of the cluster, was observed during both seasons.

In total, we collected 239 and 244 frames of the central field of $\mathrm{M} 92$ in the $I_{\mathrm{C}}$ and $V$ bands, respectively. For the second, off-centre field, 145 frames in the $I_{\mathrm{C}}$ and 119 frames in the $V$ band were obtained. On most nights the weather was very good. On some nights, however, the sky brightness was rather high due to the bright Moon. The seeing changed in a rather wide range, between 1.7 and 3.8 arcsec, with a typical value of 2.5 arcsec. Usually, the exposure times amounted to $300 \mathrm{~s}$.

The pre-processing of the frames was performed in the usual way and consisted of subtracting bias and dark frames and applying the flat-field correction. Instrumental magnitudes for all stars in the field were computed using the DAOPHOT profile-fitting software (Stetson 1987). All images were reduced in the same way as described by Jerzykiewicz et al. (1996). We identified 6371 stars in both observed fields. The finding charts for the monitored fields are shown in Figs. 1 and 2. For clarity, we show only stars brighter than 18 mag in $V$.

Our average instrumental magnitudes and colours were transformed to the standard ones using the recent photometric data of Stetson (2000). 142 standard stars in common with Stetson (2000) were chosen and the following transformation equations were obtained:

$$
\begin{aligned}
V-v & =+0.065(v-i)+15.522, & & \sigma=0.031 \\
I_{\mathrm{C}}-i & =-0.001(v-i)+14.645, & & \sigma=0.026 \\
V-I_{\mathrm{C}} & =+1.066(v-i)+00.878, & & \sigma=0.019
\end{aligned}
$$

where uppercase letters denote standard magnitudes and lowercase letters, the instrumental ones; $\sigma$ is the standard deviation from the fit.

Using the above given equations, instrumental $V$ and $I_{\mathrm{C}}$ magnitudes of the variable stars were transformed to the standard system in the following way. For each variable and both filters phased light curves were decomposed into the Fourier series with number of harmonics chosen by eye to obtain the best fit. Next, individual instrumental magnitudes were transformed to the standard ones using the colour indices defined as a difference of these two smooth 


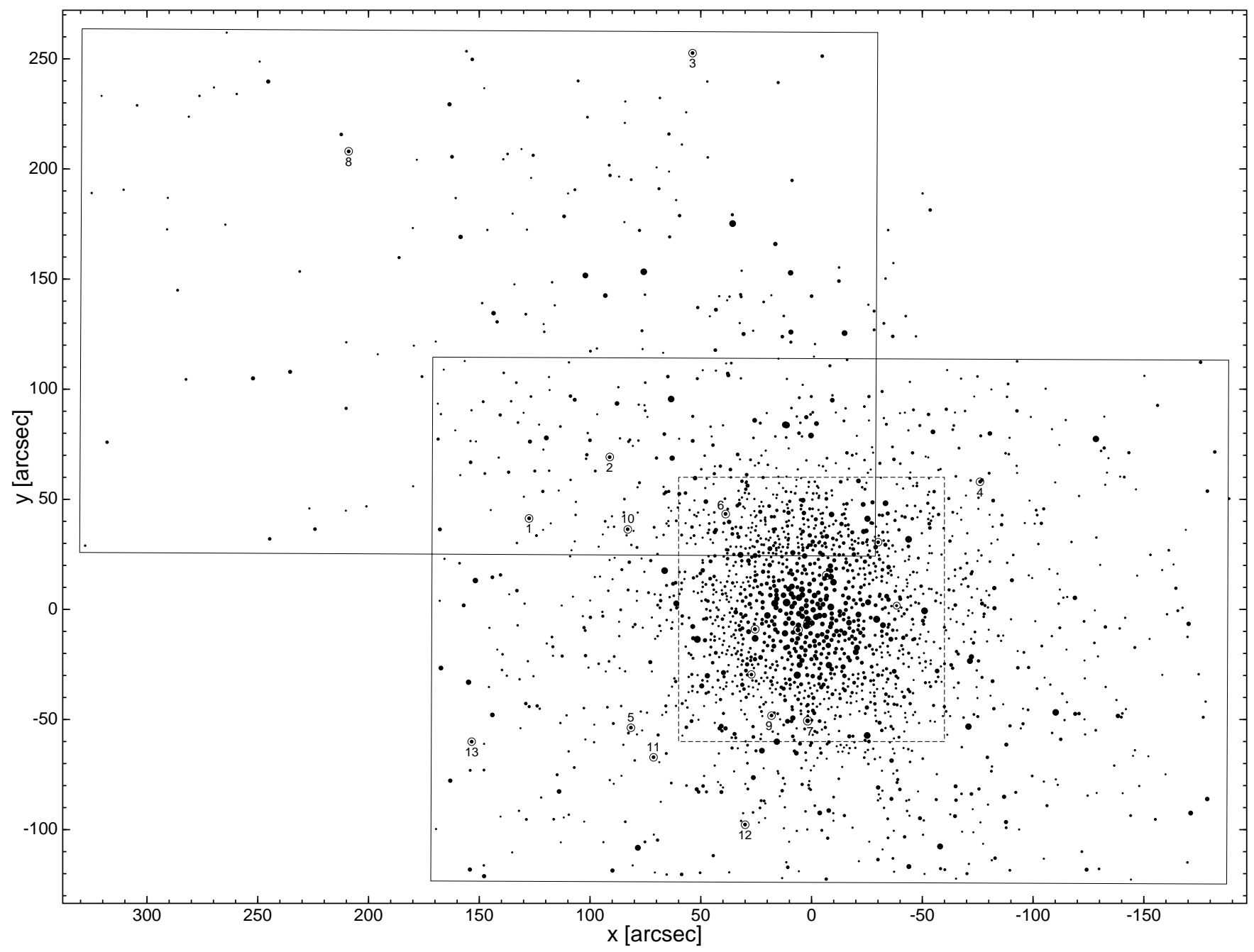

Fig. 1. Schematic view of the observed fields of M 92. Only stars brighter than 18 mag in $V$ are shown. The variable stars are enclosed in open circles. Additionally, variables lying more than 0.8 arcmin from the centre of the cluster are labeled with their numbers, given in Table 1. Enlargement of the central $2 \times 2 \operatorname{arcmin}^{2}$ area is shown in Fig. 2. Positions $(x, y)$ are in the reference frame of the CVSGC. North is up, east to the left

curves at the appropriate pulsation phase. In the case of RR Lyrae stars with Blazhko effect, the observations in both filters were divided into several groups in such a way as to obtain phase-magnitude diagrams with constant amplitude. Next, the procedure described above was applied to these groups of observations.

In order to search for variable stars in the core of the cluster we reduced our CCD frames using also the Image Subtraction Method (ISM) package developed by Alard \& Lupton (1998). This method allows obtaining very good quality light curves even in very crowded fields. The same procedure of reductions as in Kopacki (2000) was used; we refer the reader to this paper for details.

\section{Variable stars}

Out of the 28 variables and suspected variables listed in the CVSGC, only two, v14 and v16, have not been observed by us. Our observations of v12 indicate that its period was in error and we derived a correct value, which turned out to be a $1 \mathrm{~d}^{-1}$ alias of the previous one. It should be mentioned, however, that Sawyer-Hogg (1973) listed a period of about $0.41 \mathrm{~d}$ for this variable, almost the same as that obtained by us. The incorrect period of $0.29 \mathrm{~d}$ given in the CVSGC seems to come from Kukarkin \& Kukarkina (1980). The period of the small amplitude RR Lyrae variable v13 has been determined for the first time. Variable v7, until now classified as an RR Lyrae star with a period of about $0.515 \mathrm{~d}$, turned out to be of the BL Herculis type with the period approximately twice long. Already Hachenberg (1939) noticed that this star is almost a magnitude brighter than RR Lyrae stars in M 92 and thus supposed that it might be in fact a Cepheid. However, he regarded it to be the RR Lyrae variable (blended with another unresolved star) and his classification is maintained also in the CVSGC. Two suspected variables of Nassau (1938): red giant, v15, and an RR Lyrae star, v17, turned out to be constant in light. Surprisingly, only one out of the 11 suspected RR Lyrae stars of Kadla et al. (1983), viz. v25, appears to be variable. All the remaining ten 


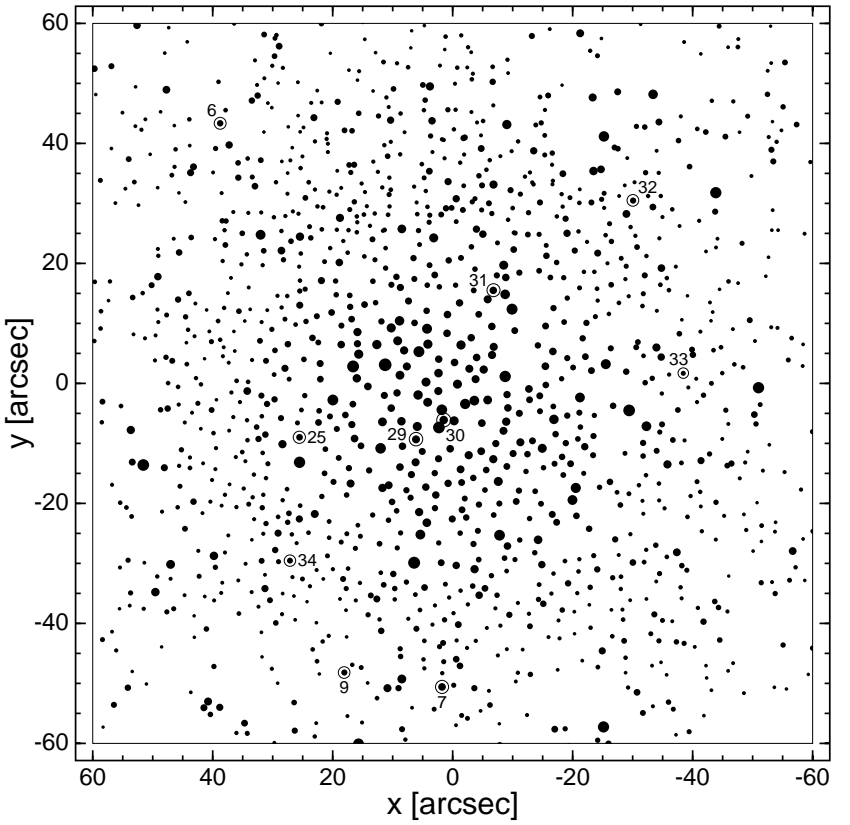

Fig. 2. Schematic view of the central $2 \times 2 \operatorname{arcmin}^{2}$ area of M 92. Only stars brighter than 18 mag in $V$ are shown. Variable stars are enclosed in open circles and labeled with their numbers given in Table 1. Positions $(x, y)$ are in the reference frame of the CVSGC. North is up, east to the left

stars show no evidence of variability. The $V$ - and $I_{\mathrm{C}}$-filter light curves of the 13 previously known RR Lyrae stars we observed are plotted in Fig. 3. The light curves of the reclassified variable $\mathrm{v} 7$ are shown in Fig. 4.

In addition, six new variable stars have been found in the very core of the cluster. Four of them are of RR Lyrae type and the remaining two of SX Phoenicis type. Extending the numbering scheme of the CVSGC for M 92, we designate these new variables as v29 through v34. Their $V$-filter light curves are shown in Fig. 5. $I_{\mathrm{C}^{-}}$ filter light curves of these new variable stars could not be determined due to the severe saturation of bright stars in the core of the cluster in the $I_{\mathrm{C}}$-filter $\mathrm{CCD}$ frames. Altogether, the light curves have been obtained for 20 variables.

Two stars, v14 and v16, both listed in the CVSGC, lie outside the observed fields. v14 has been already classified by Hachenberg (1939) as a W UMa type eclipsing binary. v16 requires additional observations to confirm its variability.

Except for v3 and v8, the periods of all observed variable stars were determined from the $O-C$ diagrams constructed on the basis of the times of maximum obtained in the two observational seasons, 1998 and 2000. Since these two seasons are separated by almost 2 years, and the typical formal error of a time of maximum is equal to about $0.0005 \mathrm{~d}$, the accuracy of the periods of the RR Lyrae variables is of the order of $0.0000005 \mathrm{~d}$. Due to the modulation of the phase of maximum, however, the period of v11 was determined with lower precision. If known, the initial values of the periods were taken from Kukarkin \& Kukarkina (1980). For variables with unknown periods the initial

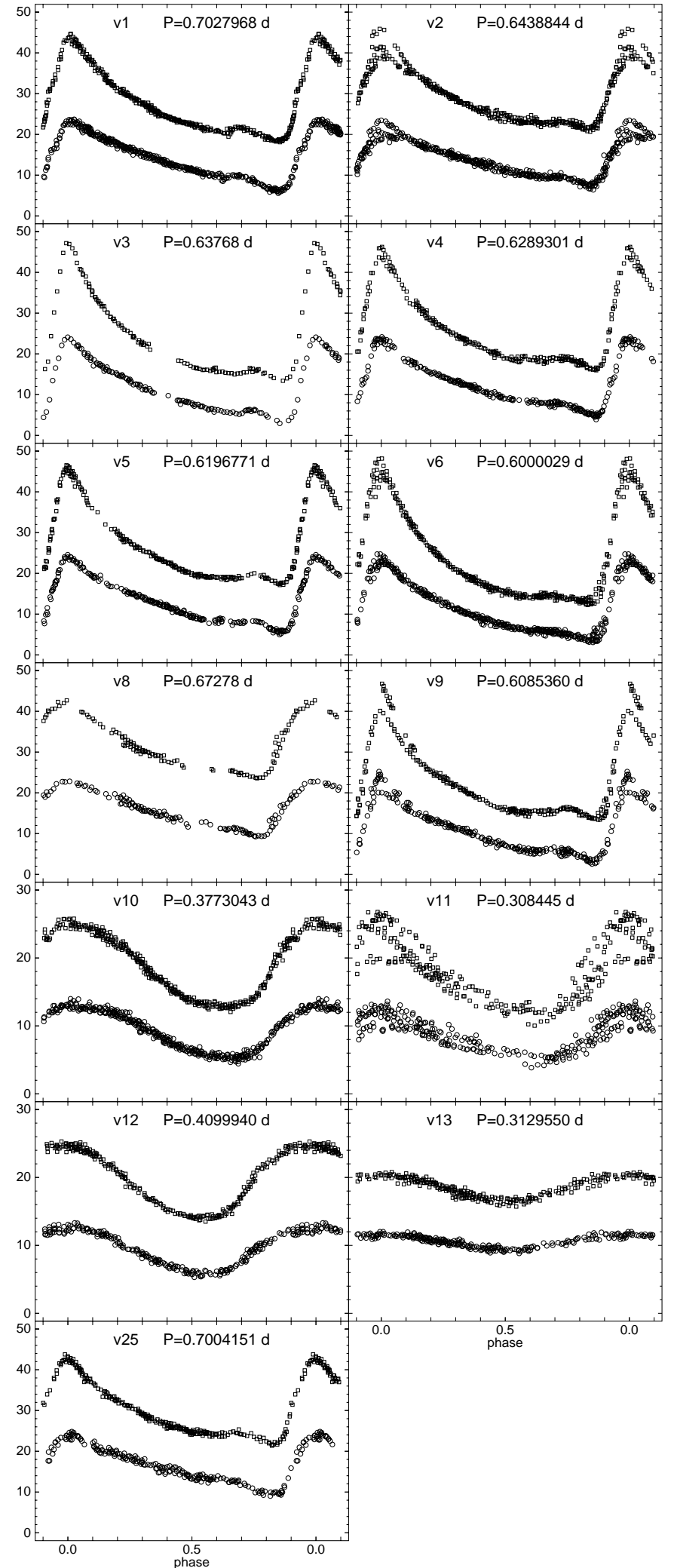

Fig. 3. $V$-filter (squares) and $I_{\mathrm{C}}$-filter (circles) light curves of the observed RR Lyrae stars listed in the CVSGC. Variables are arranged according to their numbers in that catalogue. Ordinate is expressed in arbitrary flux units. Note that scales along the ordinates are different for RRab and RRc variables 


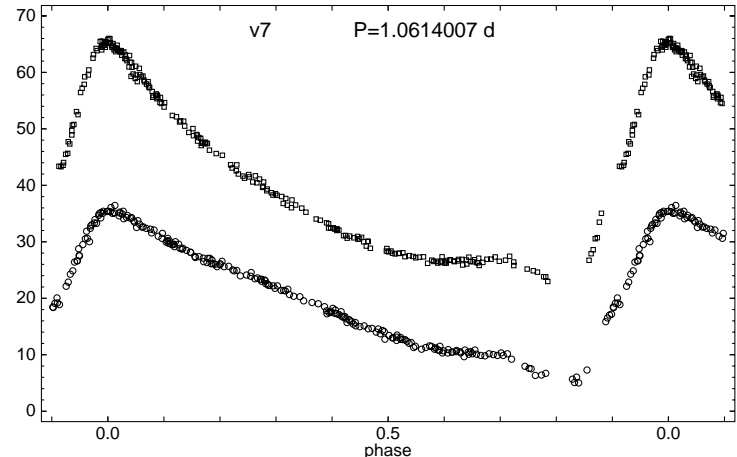

Fig. 4. $V$-filter (squares) and $I_{\mathrm{C}}$-filter (circles) light curves of the BL Herculis variable v7. Ordinate is expressed in the same units as in Fig. 3

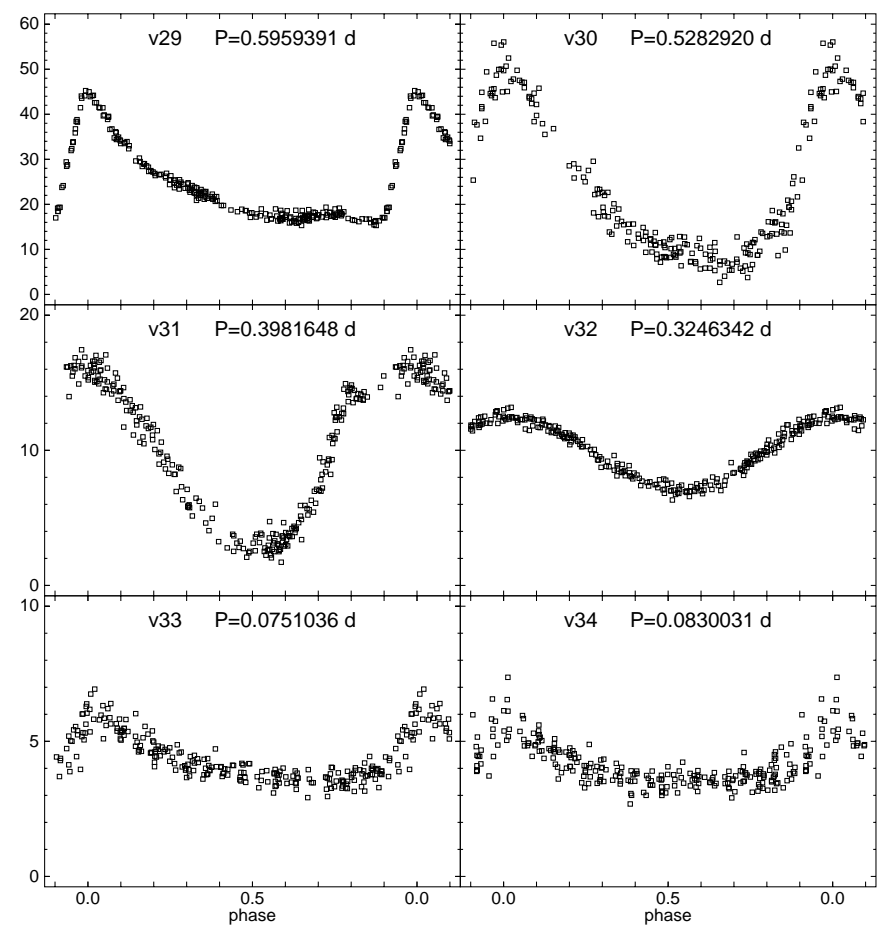

Fig. 5. $V$-filter light curves of six new variable stars: four (v29-v32) are RR Lyrae stars and two (v33 and v34), SX Phoenicis stars. Ordinate is expressed in the same units as in Fig. 3. Note the differences in the ordinate scales

period was found using multi-harmonic AoV periodogram of Schwarzenberg-Czerny (1996). The periods of v3 and v8 were derived using only the 2000 data because the observations of these two stars obtained two years earlier were very scarce. It also turned out that the 1998 observations of v3 and v8 cannot be properly phased with the 2000 data; this may be an indication of the Blazhko effect. Consequently, only the 2000 data of v3 and v8 are shown in Fig. 3.

Positions relative to the cluster centre, types of variability, and adopted periods of the stars we observed are given in Table 1. Coordinates are given in the reference frame of the CVSGC, but were redetermined using positions of 13 stars. All variables that we observed are also indicated in Figs. 1 and 2.
Table 1. Corrected positions $(x, y)$ relative to the cluster centre, types of variability, and periods $(P)$ of the variable stars in the observed field. Coordinates are given in the reference frame of the CVSGC.

\begin{tabular}{llrrl}
\hline Var & Type & $x\left[^{\prime \prime}\right]$ & $y\left[{ }^{\prime \prime}\right]$ & $P[\mathrm{~d}]$ \\
\hline v1 & RR Lyr & 127.47 & 41.35 & 0.7027968 \\
v2 & RR Lyr & 91.16 & 69.19 & 0.6438844 \\
v3 & RR Lyr & 53.68 & 252.66 & 0.63768 \\
v4 & RR Lyr & -76.07 & 58.02 & 0.6289301 \\
v5 & RR Lyr & 81.50 & -53.67 & 0.6196771 \\
v6 & RR Lyr & 38.79 & 43.29 & 0.6000029 \\
v7 & BL Her & 1.88 & -50.52 & 1.0614007 \\
v8 & RR Lyr & 208.97 & 207.98 & 0.67278 \\
v9 & RR Lyr & 18.10 & -48.17 & 0.6085360 \\
v10 & RR Lyr & 82.95 & 36.41 & 0.3773043 \\
v11 & RR Lyr & 71.29 & -67.11 & 0.308445 \\
v12 & RR Lyr & 29.91 & -97.74 & 0.4099940 \\
v13 & RR Lyr & 153.38 & -60.14 & 0.3129550 \\
v25 & RR Lyr & 25.47 & -8.95 & 0.7004151 \\
\hline v29 & RR Lyr & 6.02 & -9.33 & 0.5959391 \\
v30 & RR Lyr & 1.48 & -6.11 & 0.5282920 \\
v31 & RR Lyr & -6.57 & 15.29 & 0.3981648 \\
v32 & RR Lyr & -29.97 & 30.50 & 0.3246342 \\
v33 & SX Phe & -38.01 & 2.50 & 0.0751036 \\
v34 & SX Phe & 27.69 & -29.56 & 0.0830031 \\
\hline
\end{tabular}

The periods in Table 1 are given with an accuracy resulting from the $O-C$ diagram analysis. In most cases the periods we derived do not differ significantly from those determined earlier. Typical difference is of the order of $0.00002 \mathrm{~d}$. However, for v3 and v8 the difference is almost 20 times larger.

As can be seen in Fig. 3, three RRab variables, v2, $\mathrm{v} 6$, and v9, show cycle-to-cycle variations of the maximum brightness, a symptom of the Blazhko effect. Very pronounced light-curve modulation is also visible for one RRc type star, v11. This variable deserves more detailed analysis and will be discussed separately. CVSGC lists five RR Lyrae stars with Blazhko effect: v2, v5, v8, v10, and v11. Our observations of v5 and v10 do not show any evidence of this effect. For v8 we have only one observational season so we cannot state firmly whether this star is a Blazhko variable or not.

The total number of known RR Lyrae stars in M 92 is now equal to 17, 11 of them being of type RRab and the remaining 6 of RRc. After revision, the fraction of the RRab stars changed from about 75 per cent to 65 per cent, a value somewhat more typical for an Oosterhoff type II globular cluster. The mean period of RRab variables amounts now to $0.63 \pm 0.05 \mathrm{~d}$, while the average period for RRc variables is equal to $0.36 \pm 0.05 \mathrm{~d}$.

The light curves are available in electronic form from CDS in Strasbourg via anonymous ftp to 130.79.128.5.

Bright giants in globular clusters usually show some degree of variability. In M 53, for example, seven giants are known to be variable (Kopacki 2000). Three red giants in M 92 suspected by Walker (1955) to be variable 


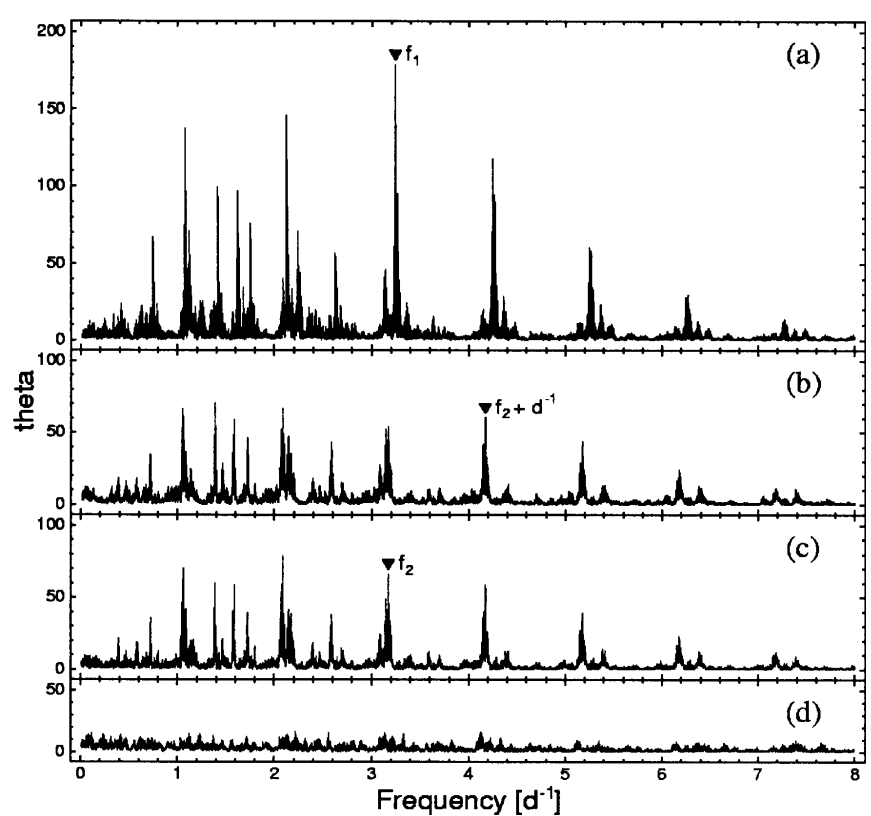

Fig. 6. AoV periodograms of the RRc variable v11: a) for original $V$-filter data, b) for $V$-filter observations after prewhitening with frequency $f_{1}=3.2421 \mathrm{~d}^{-1}$, c) for $I_{\mathrm{C}}$-filter observations after removing the same frequency, and d) for $V$-filter observations prewhitened with both $f_{1}$ and $f_{2}=3.1715 \mathrm{~d}^{-1}$

are, unfortunately, outside the fields we observed. All observed red giants were checked for variability and no variable was found. It should be noted, however, that all bright giants were heavily saturated in our CCD frames. Welty (1985) looked for variability among almost 60 red giants of M 92 lying within an annular region centred on the cluster with inner radius of 1.5 arcmin and outer radius of about 10 arcmin and found no variable star either.

\subsection{The case of $v 11$}

The only observed RRc type variable showing the lightcurve modulation is v11. We have determined several times of maximum for this star and it turned out that not only the range of brightness variations but also the phase of light maximum changes (see Fig. 3). This is an indication of the multiperiodicity. To check the possibility that v11 is multiperiodic we calculated for this variable the AoV periodogram. To account for non-sinusoidal shape of the light curve all subsequent AoV periodograms were computed with expansion into three harmonics. The periodogram of the original $V$-filter data is presented in Fig. 6a. The highest peak at frequency $f_{1}=3.2421 \mathrm{~d}^{-1}$ is clearly seen. This frequency corresponds to the period $P_{1}=0.30844 \mathrm{~d}$ which is, as expected, almost identical with the one derived from the $O-C$ analysis. The periodogram of the $I_{\mathrm{C}}$-filter observations shows maximum peak at the same frequency $f_{1}$.

Periodograms of the residuals obtained after prewhitening the observations in both filters with the main frequency $f_{1}$ and its first two harmonics are shown in Figs. 6b and c. It should be noted here that
AoV periodogram with harmonics included produces high spurious peaks at the subharmonics of the true frequency and its $1 \mathrm{~d}^{-1}$ aliases. These additional peaks are clearly seen at frequencies $f<3 \mathrm{~d}^{-1}$ in Figs. $6 \mathrm{a}-\mathrm{c}$. In the range of frequencies greater than $3 \mathrm{~d}^{-1}$ the highest peak in the spectrum for $I_{\mathrm{C}}$-filter residuals occurs at frequency $f=3.1715 \mathrm{~d}^{-1}$ (see Fig. 6c), whereas the maximum peak in the spectrum for $V$-filter residuals is seen at frequency $f+1 \mathrm{~d}^{-1}$ (see Fig. 6b). This difference is caused by slightly different distribution in time of the $V$ and $I_{\mathrm{C}}$-filter observations.

Owing to the aliasing problem we cannot state firmly which frequency, $f$ or its $1 \mathrm{~d}^{-1}$ alias, is the true one. In Fig. $6 \mathrm{~d}$ we show the periodogram of the $V$-filter residuals obtained after removing the $f_{1}$ and $f_{2}=f$ frequencies and their harmonics. Almost exactly the same residuals are obtained assuming $f_{2}=f+1 \mathrm{~d}^{-1}$. As can be seen, there is no further significant frequency in the spectrum. From the frequency analysis presented here we can only draw a conclusion that v11 is a double-period variable. It should be noted that because of the observing window, there is a possibility that the frequencies derived for v11 are in error by several times $(\Delta T)^{-1}$, where $\Delta T \approx 650 \mathrm{~d}$ is an average difference between the 1998 and 2000 observations.

Assuming $f_{2}=f+1 \mathrm{~d}^{-1}$ as a second frequency we obtain a ratio of the periods, $P_{2} / P_{1}$, equal to about 0.777 . This value does not correspond neither to an RRd variable nor to an RRe star (RR Lyrae variable pulsating simultaneously in the first and second overtone modes). Alcock et al. (2000) published recently the results of the frequency analysis of 1350 RRc variables in Large Magellanic Cloud. They found no example of biperiodic RRc variable with the ratio of periods similar to the ratio obtained for v11.

If we assume the second frequency, $f_{2}$, to be equal to $f$, we get two close frequencies with a small separation, $\Delta f=f_{1}-f_{2}$, amounting to about $0.07 \mathrm{~d}^{-1}$. In this case v11 would belong to the new group of the doublemode RRc stars recently discovered by Olech et al. (1999a, 1999b) (see also Moskalik 2000). The two frequencies of these variables, designated by Alcock et al. (2000) as RR1$v 1$ stars, are very closely spaced; the ratios of periods are higher than 0.95 and could be as large as 0.999 (Alcock et al. 2000). For ratios of this order it is clear that if $P_{1}$ is the first overtone radial mode then the second period, $P_{2}$, is a non-radial one.

\section{Light-curve parameters}

Mean brightnesses of variable stars were derived both as the intensity- and magnitude-weighted averages. The mean magnitude-weighted brightnesses, $\langle V\rangle_{\mathrm{m}}$ and $\left\langle I_{\mathrm{C}}\right\rangle_{\mathrm{m}}$, and the ranges of variability, $\Delta V$ and $\Delta I_{\mathrm{C}}$, were derived according to the formulae

$$
\begin{aligned}
\langle M\rangle_{\mathrm{m}} & =\oint f_{M}(\phi) \mathrm{d} \phi \\
\Delta M & =\max _{\phi}\left\{f_{M}(\phi)\right\}-\min _{\phi}\left\{f_{M}(\phi)\right\},
\end{aligned}
$$


Table 2. Average intensity-weighted, $\langle V\rangle_{\mathrm{i}}$, and magnitudeweighted, $\langle V\rangle_{\mathrm{m}}, V$ brightnesses, colour indices, $\left\langle V-I_{\mathrm{C}}\right\rangle_{\mathrm{i}}$ and $\left\langle V-I_{\mathrm{C}}\right\rangle_{\mathrm{m}}$, and the ranges of variability, $\Delta V$ and $\Delta I_{\mathrm{C}}$, for $12 \mathrm{RR}$ Lyrae stars and the BL Herculis variable v7 all located well outside the cluster core. Additionally, $\Delta V$ ranges, derived from the ISM photometry are shown for v25 and four new RR Lyrae stars situated in the central area of the cluster. $\sigma$ denotes standard deviation of the magnitudes from the best fit Fourier decomposition curve. $\left\langle V-I_{\mathrm{C}}\right\rangle$ colour indices are defined as a differance $\langle V\rangle-\left\langle I_{\mathrm{C}}\right\rangle$. See the text for full definitions of the parameters

\begin{tabular}{lccccccccc}
\hline Var & $\langle V\rangle_{\mathrm{i}}$ & $\langle V\rangle_{\mathrm{m}}$ & $\left\langle V-I_{\mathrm{C}}\right\rangle_{\mathrm{i}}$ & $\left\langle V-I_{\mathrm{C}}\right\rangle_{\mathrm{m}}$ & $\Delta V$ & $\Delta I_{\mathrm{C}}$ & $\sigma_{V}$ & $\sigma_{I_{\mathrm{C}}}$ \\
& {$[\mathrm{mag}]$} & {$[\mathrm{mag}]$} & {$[\mathrm{mag}]$} & {$[\mathrm{mag}]$} & {$[\mathrm{mag}]$} & {$[\mathrm{mag}]$} & {$[\mathrm{mag}]$} & {$[\mathrm{mag}]$} \\
\hline v1 & 15.02 & 15.05 & 0.50 & 0.52 & 0.86 & 0.55 & 0.02 & 0.02 \\
v2 & 15.08 & 15.11 & 0.48 & 0.50 & 0.85 & 0.55 & 0.02 & 0.02 \\
v3 & 15.09 & 15.14 & 0.48 & 0.51 & 1.17 & 0.74 & 0.01 & 0.01 \\
v4 & 15.12 & 15.16 & 0.46 & 0.47 & 0.84 & 0.67 & 0.13 & 0.17 \\
v5 & 15.12 & 15.16 & 0.47 & 0.50 & 0.98 & 0.64 & 0.02 & 0.02 \\
v6 & 14.96 & 15.02 & 0.46 & 0.49 & 1.09 & 0.71 & 0.03 & 0.02 \\
v7 & 14.15 & 14.17 & 0.54 & 0.55 & 0.64 & 0.43 & 0.02 & 0.03 \\
v8 & 15.05 & 15.07 & 0.49 & 0.50 & 0.67 & 0.47 & 0.03 & 0.02 \\
v9 & 15.13 & 15.18 & 0.51 & 0.53 & 1.19 & 0.69 & 0.04 & 0.04 \\
v10 & 15.11 & 15.13 & 0.38 & 0.39 & 0.48 & 0.30 & 0.03 & 0.02 \\
v11 & 15.10 & 15.11 & 0.29 & 0.30 & 0.62 & 0.40 & 0.05 & 0.02 \\
v12 & 15.06 & 15.07 & 0.42 & 0.42 & 0.41 & 0.25 & 0.02 & 0.02 \\
v13 & 15.13 & 15.14 & 0.30 & 0.30 & 0.15 & 0.08 & 0.02 & 0.01 \\
\hline v25 & - & - & - & - & 0.75 & - & 0.01 & - \\
v29 & - & - & - & - & 0.95 & - & 0.02 & - \\
v30 & - & - & - & - & 1.59 & - & 0.09 & - \\
v31 & - & - & - & - & 0.52 & - & 0.03 & - \\
v32 & - & - & - & - & 0.21 & - & 0.01 & - \\
\hline
\end{tabular}

where $\phi$ is the phase of the pulsation period, " $M$ " stands either for " $V$ " or " $I_{\mathrm{C}}$ ", and $f_{M}$ is a smooth curve obtained from the Fourier decomposition of the light curve of a given variable. The mean intensity-weighted brightnesses, $\langle V\rangle_{\mathrm{i}}$ and $\left\langle I_{\mathrm{C}}\right\rangle_{\mathrm{i}}$, were computed according to the formula

$$
\langle M\rangle_{\mathrm{i}}=-2.5 \log \oint g_{M}(\phi) \mathrm{d} \phi,
$$

where $g_{M}$ is a smooth curve obtained from the Fourier decomposition of the intensity curve. In the case of the stars with an obvious Blazhko effect, namely v2, v6, v9, and v11, their light curve parameters were derived from the observations which covered the epochs of the largest observed amplitude.

The light-curve parameters are given in Table 2. It should be noted that the average colours, $\left\langle V-I_{\mathrm{C}}\right\rangle$, are not derived from $\left(V-I_{\mathrm{C}}\right)$ curves. Instead, they are defined as a difference of the mean brightnesses, $\langle V\rangle$ and $\left\langle I_{\mathrm{C}}\right\rangle$. As a measure of the error of average magnitudes and colours, we show also in this table standard deviations, $\sigma_{V}$ and $\sigma_{I_{\mathrm{C}}}$, of the phased light curves in, respectively, $V$ and $I_{\mathrm{C}}$ passbands, from the best fit Fourier decomposition curves. The relatively large scatter for $\mathrm{v} 4$ is caused by blending of this star with another star of similar brightness (see Fig. 1). Due to this, the light ranges of v4 may be slightly underestimated. Since a reliable DAOPHOT profile photometry could be obtained only for the stars located well outside the cluster core, in Table 2 all defined above parameters are listed only for those variables whose distance from the cluster centre is greater than 0.8 arcmin.

The mean $V$ magnitude of the horizontal branch, $\left\langle V_{\mathrm{HB}}\right\rangle_{\mathrm{i}}$, estimated using intensity-weighted mean magnitudes of 12 RR Lyrae stars listed in Table 2 is equal to $15.08 \pm 0.01$ mag.

Perhaps the most serious drawback of the ISM of Alard \& Lupton (1998) is its unability to derive light curves expressed in magnitudes without any additional information. This is caused by the fact that in this method only a difference between variable's fluxes in two CCD frames are computed (on the other hand this is the main advantage of this method) and no information on the total flux from the star could be obtained. The conversion of the ISM light curves (expressed in arbitrary flux units) into magnitudes is possible only when at least one magnitude of the variable star is known. This magnitude can be derived using DAOPHOT package, but it does not work for stars in such dense stellar field as the core of the globular cluster.

In view of the above remarks on the ISM it seems impossible to derive ranges of variability expressed in magnitude for new variables, since all they are less than 1 arcmin distant from the cluster centre. This is true for the two new SX Phoenicis stars. In the case of new RR Lyrae stars, however, we may use the fact that all stars of this type in a given cluster have very similar mean brightness which can be estimated from the photometry of other RR Lyrae stars located well outside the cluster core.

Using several RR Lyrae stars for which both reliable DAOPHOT and ISM light curves were obtained we determined linear transformation between the flux measured by DAOPHOT and differential flux of the ISM. Assuming that all new RR Lyrae stars have the same intensityweighted average brightness equal to the average magnitude of the horizontal branch, $\left\langle V_{\mathrm{HB}}\right\rangle_{\mathrm{i}}$, we were able to compute $\Delta V$ ranges of these stars. Derived in this way $\Delta V$ ranges of RR Lyrae variables v25, v29, v30, v31, and v32, lying in the core of M 92, are listed in Table 2.

\section{Conclusions}

The colour-magnitude diagram for M 92 is shown in Fig. 7. Only stars with distance from cluster centre greater than 1.5 arcmin and error in colour smaller than $0.2 \mathrm{mag}$ are plotted. Variable stars are represented by their intensity-weighted mean $\langle V\rangle_{\mathrm{i}}$ brightnesses and magnitude-weighted mean $\left\langle V-I_{\mathrm{C}}\right\rangle_{\mathrm{m}}$ colour indices. The choice of the intensity-weighted average magnitude is a logical consequence of the fact that the mean brightness should be a measure of the mean luminosity of a variable star. We use magnitude-weighted colour in accordance with Sandage (1990) who pointed out that a magnitude average is a more reliable temperature indicator.

The colour-magnitude diagram shows a well-defined red giants branch, asymptotic giant branch, and a horizontal branch. Figure 8 presents a more detailed view of 


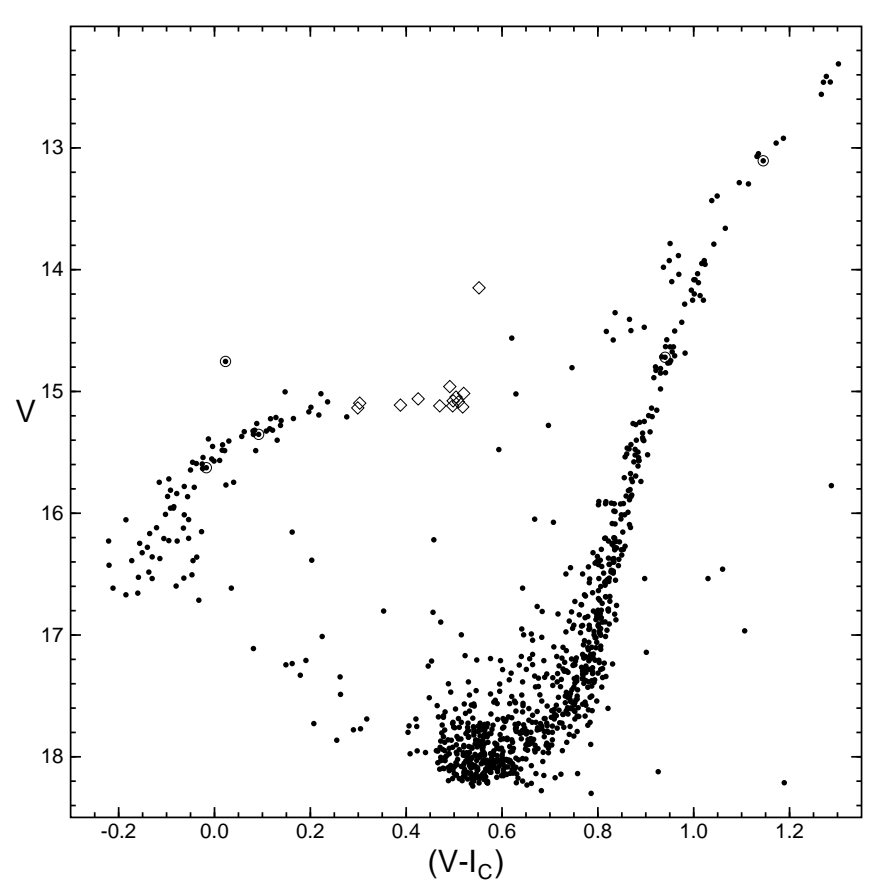

Fig. 7. The colour-magnitude diagram for M 92. Only stars with the distance from cluster centre greater than 1.5 arcmin and the error in $\left(V-I_{\mathrm{C}}\right)$ smaller than $0.2 \mathrm{mag}$ are included. Variable stars are shown with open diamonds and are represented by their intensity-weighted mean $V$ brightness and magnitude-weighted mean $\left(V-I_{\mathrm{C}}\right)$ colour index (see the text for definitions). Five suspected variable stars listed in the CVSGC, which turned out to be constant, are also shown with symbols enclosed within circles

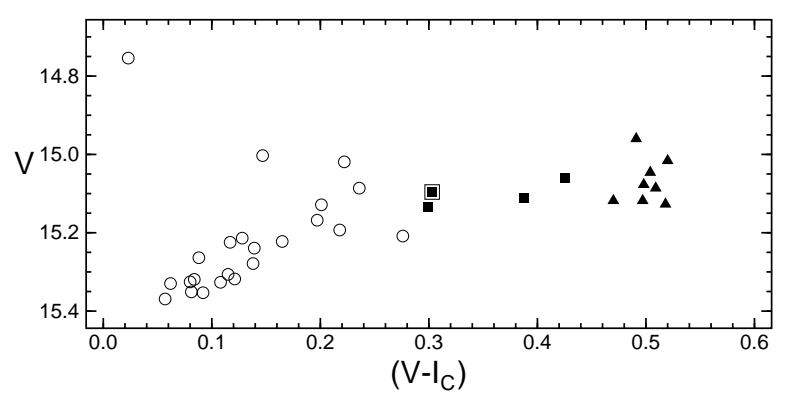

Fig. 8. The colour-magnitude diagram for the horizontal branch of M 92. The RRab stars are shown with filled triangles and the RRc stars with filled squares. Additionally, biperiodic $R R c$ variable $v 11$ is indicated with symbol enclosed within open square. Non-variable stars are shown with open circles

the horizontal branch. As we can see in this figure, there is a clear separation between the variable and constant stars and also between the RRab and RRc variables.

In Fig. 8 the biperiodic non-radial pulsating RRc variable v11 is situated at the blue edge of the instability strip. This is in accordance with the recent results obtained by Olech et al. (1999a) for three non-radial pulsating RRc stars in M 55 and Olech et al. (2000) in the case of also three RRc variables of this subtype in the cluster NGC 6362.

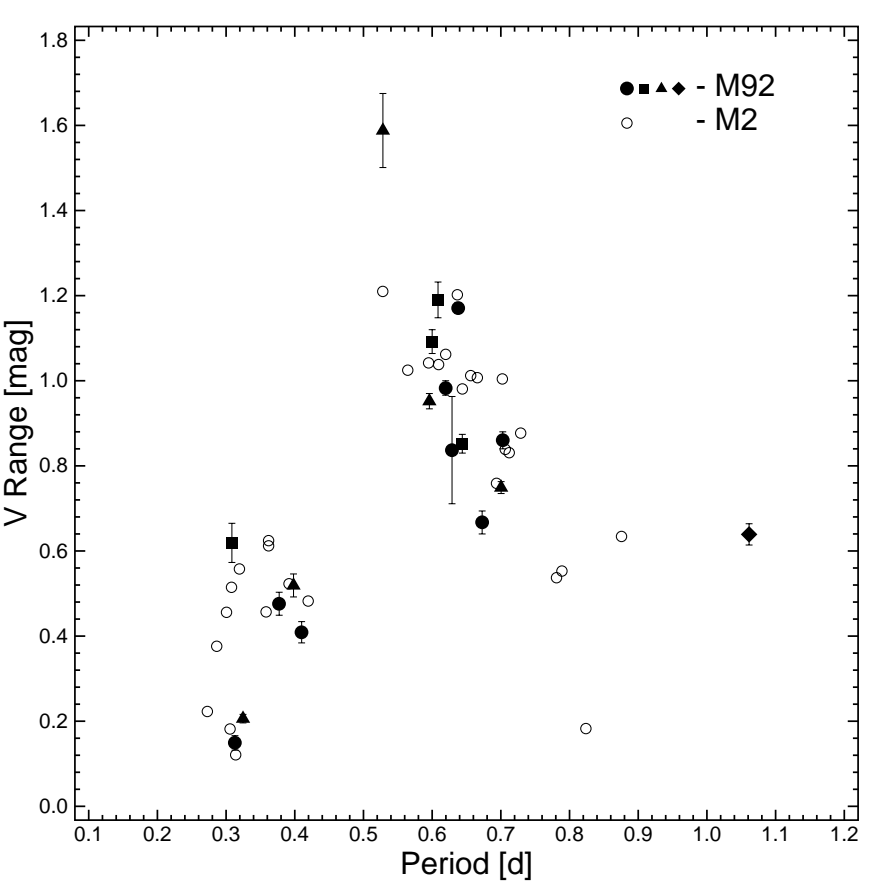

Fig. 9. $V$-filter ranges as a function of period for the RR Lyrae stars (filled circles and squares) and the BL Herculis variable (filled diamond) in M 92. Blazhko variables are indicated with filled squares. RR Lyrae stars whose $\Delta V$ ranges were determined indirectly from the ISM light curves (see the text for explanations) are shown with filled triangles. The M 2 RR Lyrae stars (open circles) are also shown for comparison.

Five suspected variable stars from the CVSGC, which turned out to be constant in light and for which reliable photometry was obtained are also shown in Fig. 7. Two of them, v18 and v19, occupy the blue part of the horizontal branch and lie outside the RR Lyrae instability strip, v26 seems to be a field star. The remaining two, v15 and v17, are red giants.

In the colour-magnitude diagram the BL Herculis variable v7 lies $0.93 \mathrm{mag}$ above the horizontal branch, and therefore its brightness is in the range expected for a cluster member. Since the question of the correct metallicityluminosity relation for RR Lyrae stars is not satisfactorily answered yet, we assumed for the M 92 RR Lyrae stars an absolute magnitude $M_{V}(\mathrm{RR})=0.45 \mathrm{mag}$ derived by Storm et al. (1994) from a Baade-Wesselink analysis of two RR Lyrae variables in the cluster. Using this value we get the absolute magnitude of v7 equal to about $-0.5 \mathrm{mag}$. This result, together with the period of v7 fits within the uncertainities the period-luminosity relation defined by 40 other Population II Cepheids known in globular clusters (Nemec et al. 1994).

Figure 9 shows $V$-filter ranges as a function of period for RR Lyrae stars in M 92 listed in Table 2. The BL Herculis variable, v7, is also included. The relation for the M 2 RR Lyrae stars is also shown for comparison. The data for M 2 were taken from Lee \& Carney (1999).

The new RRab star v30 with the largest range of variability (and the smallest period among RRab stars in 
M 92) fits very well the sequence of other RRab variables. The relatively large error of the light range for this variable is caused by the fact that it is situated almost in the centre of a clump of three bright stars, as can be seen in Fig. 2 (see also the light curve in Fig. 5).

Period-shift analysis for M 92 and M 2 has been performed. Fitting the M 92 data with the slope of the M 2 data produced negligible period shift of $0.004 \pm 0.011$. However, linear relation between logarithm of period and amplitude for the M 2 RRab variables has been determined using only 8 stars showing no evidence of Blazhko effect. Despite significant difference in the metal abundance between these two clusters $([\mathrm{Fe} / \mathrm{H}]=-2.24$ for M 92 and -1.62 for M 2) there is no firm evidence for a shift in period. It should be noted, however, that both M 92 and M 2 belong to the same Oosterhoff group. Very small period shift of $0.014 \pm 0.010$ has been also obtained from comparison of M 92 with M 53, both of them having similar metallicity $([\mathrm{Fe} / \mathrm{H}]=-2.04$ for $\mathrm{M} 53)$. The data for M 53 were taken from Kopacki $(2000,2001)$. They included almost three times more RRab stars than in M 2.

Concluding, the results we obtained strengthen the statement that the period shift is not a continuous function of the metal abundance as proposed by Sandage (1981), but is rather dependent on the Oosterhoff type. This is in accordance with recent results of the comparison of period-amplitude relations of several globular clusters done by Clement \& Shelton (1999) and Clement (2000).

Acknowledgements. I want to express here my gratitude to A. Pigulski for his very kind help in preparation of this paper. I thank Prof. M. Jerzykiewicz for critical reading the manuscript and Z. Kołaczkowski for assistance during the observations and stimulating discussions. I am also grateful to the referee, Dr. C. Clement for valuable comments. This work was supported by KBN grant No. 2 P03D 00619.

\section{References}

Alard, C., \& Lupton, R. H. 1998, ApJ, 503, 325

Alcock, C., Allsman, R., Alves, D. R., et al. 2000, ApJ, 542, 257

Arp, H. C., Baum, W. A., \& Sandage, A. R. 1953, AJ, 58, 4

Bartolini, C., Battistini, P., \& Nasi, E. 1968, Bologna Publ. IX, No. 15,3

Blazhko, S. 1907, Astron. Nachr., 175, 325

Carney, B. W., Storm, J., Trammell, S. R., \& Jones, R. V. 1992, PASP, 104, 44

Clement, C. 1997, Preliminary Fourth Edition of the Catalogue of Variable Stars in Globular Clusters, electronic version

Clement, C. 2000, PASPC, 203, 266
Clement, M. C., \& Shelton, I. 1999, ApJ, 515, L85

Cohen, J. G. 1992, ApJ, 400, 528

Cohen, J. G., \& Matthews, K. 1992, PASP, 104, 1205

Grundahl, F., VandenBerg, D. A., Bell, R. A., et al. 2000, AJ, 120,1884

Guthnick, P., \& Prager, R. 1925, Sitz. d. Preuss, Akad. d. Wiss., 27, 508

Hachenberg, O. 1939, Z. Astrophys., 18, 49

Jerzykiewicz, M., Pigulski, A., Kopacki, G., Miałkowska, A, \& Niczyporuk, S. 1996, Acta Astron., 46, 253

Kadla, Z. I., Yablokova, N. V., Gerashchenko, A. N., \& Spasova, N. 1983, Peremennye Zvezdy, 21, 827

Kheylo, E. S. 1964, IBVS, 43

Kheylo, E. S. 1965, IBVS, 104

Kopacki, G. 2000, A\&A, 358, 547

Kopacki, G. 2001, in preparation

Kopacki, G., \& Pigulski, A. 1995, Acta Astron., 45, 753

Kovács, G., Alcock, C., Allsman, R., et al. 2000, PASPC, 203, 313

Kukarkin, B. V., \& Kukarkina, N. P. 1980, Peremennye Zvezdy, 21,365

Lee, J. W., \& Carney, B. W. 1999, AJ, 117, 2868

Moskalik, P. 2000, PASPC, 203, 315

Nassau, J. J. 1938, ApJ, 87, 361

Nemec, J. M, Linnell-Nemec, A. F., \& Lutz, T. E. 1994, AJ, 108,222

Olech, A., Kałużny, J., Thompson, I. B., et al. 1999a, AJ, 118, 442

Olech, A., Woźniak, P. R., Alard, C., et al. 1999b, MNRAS, 310,759

Olech, A., Kałużny, J., Thompson, I. B., et al. 2000, submitted to MNRAS, [astro-ph/0005589]

Oosterhoff, P. Th. 1944, Bull. Astron. Inst. Netherl., X, 55

Rosenberg, A., Aparicio, A., Saviane, I., \& Piotto, G. 2000, A\&AS, 145, 451

Sandage, A. 1981, ApJ, 248, 161

Sandage, A. 1990, ApJ, 350, 603

Sawyer-Hogg, H. 1973, Publ. David Dunlop Obs., 3, No. 3

Schlegel, D. J., Finkbeiner, D. P., \& Davis, M. 1998, ApJ, 500, 525

Schwarzenberg-Czerny, A. 1996, ApJ, 460, L107

Shapley, H. 1922, Harvard Bull., No. 773

Shara, M. M., Bergeron, L. E., \& Moffat, A. F. J. 1994, ApJ, 429, 767

Stetson, P. B. 1987, PASP, 99, 191

Stetson, P. B. 2000, PASP, 112, 925

Storm, J., Carney, B. W., \& Latham, D. W. 1992a, PASP, 104, 159

Storm, J., Carney, B. W., \& Latham, D. W. 1994, A\&A, 290, 443

Storm, J., Carney, B. W., Latham, D. W., Davis, R. J., \& Laird, J. B. 1992b, PASP, 104, 168

Walker, M. F. 1955, AJ, 60, 197

Welty, D. E. 1985, AJ, 90, 2555

Zinn, R. J. 1985, AJ, 293, 424 